\title{
Risk Stratification in Cancer Patients with Acute Upper Gastrointestinal Bleeding: Comparison of Glasgow-Blatchford, Rockall and AIMS65, and Development of a New Scoring System
}

\author{
Matheus Cavalcante Franco', Sunguk Jang ${ }^{2}$, Bruno da Costa Martins ${ }^{3}$, Tyler Stevens ${ }^{2}$, Vipul Jairath ${ }^{4}$, Rocio Lopez ${ }^{2}$, John J. Vargo ${ }^{2}$, \\ Alan Barkun ${ }^{5}$, and Fauze Maluf-Filho ${ }^{3}$ \\ ${ }^{1}$ Hospital Sírio-Libanês, Brasília, Distrito Federal, Brazil, ${ }^{2}$ Department of Gastroenterology and Hepatology, Cleveland Clinic, Cleveland, \\ Ohio, USA, ${ }^{3}$ Endoscopy Unit, Cancer Institute of the University of São Paulo, São Paulo, Brazil, ${ }^{4}$ Western University, London, ON, \\ ${ }^{5}$ Division of Gastroenterology, McGill University and the McGill University Health Centre, Montreal, QC, Canada
}

Background/Aims: Few studies have measured the accuracy of prognostic scores for upper gastrointestinal bleeding (UGIB) among cancer patients. Thereby, we compared the prognostic scores for predicting major outcomes in cancer patients with UGIB. Secondarily, we developed a new model to detect patients who might require hemostatic care.

Methods: A prospective research was performed in a tertiary hospital by enrolling cancer patients admitted with UGIB. Clinical and endoscopic findings were obtained through a prospective database. Multiple logistic regression analysis was performed to gauge the power of each score.

Results: From April 2015 to May 2016, 243 patients met the inclusion criteria. The AIMS65 (area under the curve [AUC] 0.85) best predicted intensive care unit admission, while the Glasgow-Blatchford score best predicted blood transfusion (AUC 0.82 ) and the low-risk group (AUC 0.92). All scores failed to predict hemostatic therapy and rebleeding. The new score was superior (AUC 0.74 ) in predicting hemostatic therapy. The AIMS65 (AUC 0.84) best predicted in-hospital mortality.

Conclusions: The scoring systems for prognostication were validated in the group of cancer patients with UGIB. A new score was developed to predict hemostatic therapy. Following this result, future prospective research should be performed to validate the new score. Clin Endosc 2022;55:240-247

Key Words: Cancers; Gastrointestinal; Hemorrhage; In-hospital mortality; Prognostic factors

\section{INTRODUCTION}

In a recent series of patients with cancer at the Cancer Institute of Sao Paulo, we found that tumor bleeding was the main cause of upper gastrointestinal bleeding (UGIB; 23.8\%), followed by varices $(19.7 \%)$ and ulcers $(16.3 \%){ }^{1}$ The rates of

Received: March 28, 2021 Revised: June 27, 2021

Accepted: August 29, 2021

Correspondence: Matheus Cavalcante Franco

SCES trecho 4, Brasília - DF 70200-004, Brazil

Tel: +55-61-99913-2032, Fax: +55-61-99913-2032, E-mail: mcavalcantefranco@ gmail.com

ORCID: https://orcid.org/0000-0003-0525-8003

(c) This is an Open Access article distributed under the terms of the Creative Commons Attribution Non-Commercial License (http://creativecommons.org/ licenses/by-nc/3.0) which permits unrestricted non-commercial use, distribution, and reproduction in any medium, provided the original work is properly cited. mortality from UGIB in the general population ranges from $3.5 \%$ to $20 \%,^{2,3}$ and it is even higher in cancer patients presenting with overt UGIB, approaching $45 \%{ }^{1}$

It is recommended that patients with UGIB should be stratified into low or high risk of needing early hospital-based intervention and death. ${ }^{4,5}$ For that purpose, prognostic scores, which take into consideration clinical and endoscopic findings, are employed.

The Rockall score (RS) was primarily designed to assess the 30-day mortality after an episode of UGIB. ${ }^{6}$ Patients with a total RS $\leq 2$ are considered at low risk. ${ }^{7}$ Similarly, the Glasgow-Blatchford score (GBS) was developed to predict the 30-day mortality and need for intervention. The advantage of GBS is that endoscopic findings are not a part of the scoring criteria, allowing for a more rapid risk assessment. Patients with GBS $\geq 1$ are considered high-risk. ${ }^{8}$ A relatively new 
scoring system, known as AIMS65, was developed to predict in-hospital mortality using fewer clinical parameters. ${ }^{9}$ A cutoff score $\geq 2$ was identified to best predict the high-risk group. ${ }^{10}$

Patients with cancer who present with UGIB pose unique challenges due to their underlying malignancies and the consequences of medical and oncologic treatment that affect the normal response to acute UGIB. Few studies have evaluated UGIB prognostication in patients with cancer. ${ }^{1,11-13}$

This study primarily aimed to compare the performance of GBS, RS, and AIMS65 in predicting clinical outcomes, including the need for intensive care unit (ICU) admission, blood transfusion, hemostatic therapy, rebleeding, in-hospital mortality, and identifying low-risk cases who can be treated safely as outpatients in a group of patients with cancer presenting with an acute episode of UGIB. The secondary aim was to develop a pre-endoscopic model to identify cancer patients with UGIB who may need hemostatic therapy.

\section{MATERIALS AND METHODS}

\section{Study design}

This was a single-center, prospective cohort study of consecutive patients with cancer admitted to the Cancer Institute of the University of São Paulo with overt UGIB. This study was approved by the University of São Paulo Review Board (July 28, 2014; number: 229/14) and registered in the Clinical Trials database (NCT02508883). Written informed consent was obtained from each patient included in the study. This study protocol conforms to the ethical guidelines of the 1975 Declaration of Helsinki as reflected in a priori approval by the institution's human research committee.

Inclusion criteria included a confirmed diagnosis of malignant neoplasm of any site (inside or outside of gastrointestinal [GI] tract) and clinical evidence of UGIB (hematemesis or melena) within $48 \mathrm{~h}$ of presentation. Patients with hematochezia due to a proven site of bleeding in the upper GI tract were also included. Exclusion criteria were as follows: age $<18$ years, suspicion or confirmation of pregnancy, no evidence of malignant neoplasm after curative surgery or oncologic therapy, refusal to provide data for the study, incomplete data, and being lost to follow up.

\section{Treatment protocol}

Hemotransfusion was recommended by the attending medical staff in patients with serum hemoglobin below $8 \mathrm{~g} / \mathrm{dL}^{5}$

Esophagogastroduodenoscopy (EGD) was performed to confirm the presence of recent or ongoing UGIB after hemodynamic resuscitation. Patients with suspected non-variceal bleeding received intravenous proton pump inhibitors before the EGD, while those with cirrhosis and variceal UGIB received prophylactic antibiotics and terlipressin. ${ }^{4}$

UGIB was confirmed if EGD showed a malignant or benign lesion with the following bleeding stigmata: active bleeding (spurting or oozing), recent hemorrhage (visible vessel or adherent clot), and bright red or "coffee grounds in the stomach.

Endoscopic hemostasis was performed as follows: banding for esophageal varices, cyanoacrylate for gastric varices, and combined therapy (injection and hemoclip or thermal method) for peptic ulcers. ${ }^{4}$ Patients suffering from tumor bleeding not deemed treatable with endoscopy were referred for radiation therapy, angiographic embolization, or surgery. When performed, endoscopic hemostasis for tumoral bleeding was realized with either coagulation or clips, associated or not with injection. ${ }^{111}$

Rebleeding was defined as a new episode of hemorrhage after successful initial hemostasis. ${ }^{14} \mathrm{~A}$ second attempt at endoscopic hemostasis may be performed in the presence of rebleeding. When endoscopy failed to control the bleeding, the patients were referred for arterial embolization or surgery. 1,4,5

\section{Data collection}

The research team collected data on the patients' medical history, laboratory tests, and endoscopy results, which were gathered prospectively from the endoscopy database into a dedicated registry. The patients were monitored for the pertinent outcomes on the $3^{\text {rd }}, 7^{\text {th }}$, and $30^{\text {th }}$ day following initial presentation and on the day of discharge if they were admitted longer than 30 days. Patients who were discharged early (less than 30 days) were contacted via telephone if their medical records lacked the required information.

\section{Statistical analysis}

Continuous variables were analyzed by nonparametric test and student $t$-test, and categorical variables were analyzed with the chi-square test or Fisher's exact test, depending on the number of observations.

Receiver operating characteristics (ROC) analysis was performed to assess the value of the different scores in predicting outcomes. The areas under the ROC curve (AUC) and their corresponding 95\% confidence intervals (CI) are presented. DeLong's method was used to compare the AUC between scores, ${ }^{15}$ and Youden's index was utilized to determine optimal cut points. ${ }^{16}$ In addition, logistic regression analysis was used to develop a new scoring system. An automated stepwise variable selection method performed on 1,000 bootstrap samples was used to determine the final model. A $p<0.05$ was considered statistically significant. SAS (version 9.4; The SAS Institute, Cary, NC, USA) was used for all analyses. 


\section{RESULTS}

From April 2015 to May 2016, 394 consecutive patients were assessed, and 259 of them met the inclusion criteria and were included in the analysis. Sixteen patients were excluded due to the lack of data or being lost to follow-up. In total, 243 patients were considered in the final analysis. Additional demographic characteristics are listed in Table 1. All included patients underwent an EGD. The median follow-up period was 30 days

Table 1. Demographic and Clinical Characteristics

\begin{tabular}{|c|c|}
\hline & Total $(n=243)$ \\
\hline Factor & Statistics \\
\hline Age (years) & $60.6 \pm 13.6$ \\
\hline \multicolumn{2}{|l|}{ Gender } \\
\hline Female/Male & $71(29.2) / 172(70.8)$ \\
\hline \multicolumn{2}{|l|}{ Patient status } \\
\hline Outpatient & $178(73.3)$ \\
\hline Inpatient & $65(26.7)$ \\
\hline Tumor in upper GI tract & $106(43.6)$ \\
\hline \multicolumn{2}{|l|}{ Cancer stage } \\
\hline I or II & $17(7.0)$ \\
\hline III & $48(19.8)$ \\
\hline IV & $177(73.1)$ \\
\hline \multicolumn{2}{|l|}{ History } \\
\hline Hematemesis & $135(55.6)$ \\
\hline Melena & $104(42.8)$ \\
\hline Liver disease & $104(42.8)$ \\
\hline Hemoglobin (g/dL) & $8.1 \pm 2.9$ \\
\hline Albumin (g/dL) & $2.8 \pm 0.75$ \\
\hline INR & $1.4 \pm 0.53$ \\
\hline Rebleeding & $24(9.9)$ \\
\hline $\mathrm{RBC}$ transfusion & $147(60.5)$ \\
\hline ICU admission & $107(44.0)$ \\
\hline Hemostatic therapy & $104(42.8)$ \\
\hline 30-day mortality & $66(27.2)$ \\
\hline Clinical Rockall score & $5[0-7]$ \\
\hline Total Rockall score & $7[0-10]$ \\
\hline AIMS65 score & $2[0-5]$ \\
\hline Glasgow-Blatchford score & $12[0-20]$ \\
\hline
\end{tabular}

Data are presented as mean $\pm \mathrm{SD}$, number (\%), or median [range].

GI, gastrointestinal; ICU, intensive care unit; INR, international normalized ratio; RBC, red blood cell; SD, standard deviation. (range 22-30 days).

Bleeding was endoscopically confirmed in 132 (54.3\%) patients, and the most frequent bleeding sources were: primary cancer in $26.5 \%(35 / 132)$, varices in $25.7 \%$ (34/132), ulcers in $22 \%(29 / 132)$, metastasis in $13.6 \%(18 / 132)$, esophagitis in $6 \%$ $(8 / 132)$, angioectasias in $3 \%(4 / 132)$, gastroduodenal erosions in $1.5 \%(2 / 132)$, and other etiology in $1.7 \%$. In the group with upper GI cancers (primary or metastasis) and confirmed bleeding on endoscopy, the tumor was the source in $82.9 \%$ (34/41).

\section{Comparative analysis of scoring systems}

\section{A. ICU admission}

ICU admission was indicated in 44\% (107/243) of cases. The AIMS65 score was significantly superior in predicting ICU admission compared to the other scores (Table 2). An AIMS65 score $\geq 2$ maximized the sensitivity (84.1\%) and specificity (72.8\%).

A subset analysis of the group with upper GI cancers confirmed that the AIMS65 score best predicted the need for ICU admission compared to the other scores (Table 2).

\section{B. Blood transfusion}

Blood transfusion was indicated in $60.5 \%$ (147/243) of the patients. The GBS was significantly better in predicting blood transfusion compared to the other scores (Table 2), with GBS $\geq 12$ resulting in the maximal sensitivity $(70.7 \%)$ and specificity $(80 \%)$.

\section{Hemostatic therapy}

The use of hemostatic therapy was examined separately amongst patients with cancer within and outside the GI tract. In the cases with primary cancer outside the upper GI tract, hemostatic therapy was provided in $40.8 \%$ using endoscopic techniques $(85.5 \%)$, radiation (13\%), and angiographic embolization (1.5\%). In the patients with upper GI cancer, hemostatic therapy was provided for $47.3 \%$ of patients using radiation (71.4\%), endoscopic (14.3\%), surgical (6\%), and angiographic $(5.7 \%)$ techniques.

The scoring systems showed comparable results as they all poorly predicted pre-endoscopic hemostatic therapy (Table 2). Similar results were observed in the subset analysis of the cases with upper GI cancer and primary cancer outside the upper GI tract.

\section{Rebleeding}

Overall, the rebleeding rate was $9.9 \%$ (24/243), with a median of 6 days (range 1-25 days) to onset. All the scoring systems poorly predicted rebleeding (Table 2 ). 
Table 2. Discriminative Ability of Evaluated Scoring Systems

\begin{tabular}{|c|c|c|}
\hline $\begin{array}{c}\text { Outcome by } \\
\text { scoring system }\end{array}$ & $\operatorname{AUC}(95 \% \mathrm{CI})$ & $p$-value \\
\hline \multicolumn{3}{|l|}{ ICU: } \\
\hline AIMS65 & $0.85(0.80,0.89)$ & Best performance \\
\hline Glasgow-Blatchford & $0.79(0.73,0.84)$ & 0.04 \\
\hline Total Rockall & $0.71(0.65,0.77)$ & $<0.001$ \\
\hline Clinical Rockall & $0.66(0.59,0.72)$ & $<0.001$ \\
\hline \multicolumn{3}{|c|}{ ICU - Upper GI cancer group: } \\
\hline AIMS65 & $0.90(0.85,0.96)$ & Best performance \\
\hline Glasgow-Blatchford & $0.76(0.67,0.86)$ & 0.04 \\
\hline Total Rockall & $0.69(0.60,0.79)$ & $<0.001$ \\
\hline Clinical Rockall & $0.65(0.56,0.75)$ & $<0.001$ \\
\hline \multicolumn{3}{|l|}{ Blood transfusion: } \\
\hline Glasgow-Blatchford & $0.82(0.77,0.88)$ & Best performance \\
\hline AIMS65 & $0.76(0.71,0.82)$ & 0.04 \\
\hline Total Rockall & $0.73(0.67,0.79)$ & 0.005 \\
\hline Clinical Rockall & $0.67(0.60,0.74)$ & $<0.001$ \\
\hline \multicolumn{3}{|l|}{ Hemostatic therapy: } \\
\hline Glasgow-Blatchford & $0.67(0.60,0.74)$ & ns \\
\hline AIMS65 & $0.64(0.57,0.70)$ & ns \\
\hline Clinical Rockall & $0.57(0.50,0.64)$ & ns \\
\hline \multicolumn{3}{|l|}{ Rebleeding: } \\
\hline AIMS65 & $0.60(0.54,0.66)$ & ns \\
\hline Glasgow-Blatchford & $0.61(0.54,0.67)$ & ns \\
\hline Clinical Rockall & $0.62(0.55,0.68)$ & ns \\
\hline Total Rockall & $0.54(0.47,0.60)$ & ns \\
\hline
\end{tabular}

Rebleeding was observed in $10.8 \%(11 / 102)$ of cases with upper GI cancer and in 9.3\% (13/141) of cases with cancer outside the GI tract. A similar poor discriminative prediction for rebleeding was observed for all the scoring systems in the subset analysis of these patient subgroups.

\section{E. Mortality}

The overall 30 -day mortality was $27.2 \%(66 / 243)$, and it was significantly higher in the cases with confirmed bleeding at index EGD compared to the cases with no signal of active or recent bleeding during EGD (34.1\% vs. $18.9 \%$, respectively; $p=0.008$ ). The 30 -day mortality rate in the patients with confirmed bleeding at index EGD was similar regardless of whether the bleeding was caused by a tumor or not (38.1\% vs. $31.9 \%$, respectively; $p=0.46$ )
Table 2. (Continued)

\begin{tabular}{lcc}
\hline \multicolumn{1}{c}{$\begin{array}{c}\text { Outcome by } \\
\text { scoring system }\end{array}$} & AUC $(95 \%$ CI $)$ & $p$-value \\
\hline In-hospital mortality & Upper GI cancer group: \\
AIMS65 & $0.86(0.78,0.92)$ & Best performance \\
Glasgow-Blatchford & $0.69(0.60,0.78)$ & 0.003 \\
Total Rockall & $0.69(0.59,0.78)$ & 0.005 \\
Clinical Rockall & $0.64(0.54,0.73)$ & $<0.001$ \\
UGIB-related in-hospital mortality: & \\
AIMS65 & $0.86(0.81,0.96)$ & Best performance \\
Glasgow-Blatchford & $0.77(0.68,0.85)$ & 0.049 \\
Total Rockall & $0.76(0.70,0.83)$ & 0.012 \\
Clinical Rockall & $0.65(0.58,0.73)$ & $<0.001$ \\
Low-risk group: & \\
Glasgow-Blatchford & $0.92(0.88,0.95)$ & Best performance \\
AIMS65 & $0.83(0.78,0.88)$ & 0.001 \\
Clinical Rockall & $0.70(0.64,0.76)$ & $<0.001$ \\
New score - hemostatic therapy: & $0.74(0.67,0.80)$ & Best performance \\
New score & $0.67(0.60,0.74)$ & 0.027 \\
Glasgow-Blatchford & $0.64(0.57,0.70)$ & 0.001 \\
AIMS65 & $0.57(0.50,0.64)$ & $<0.001$ \\
Clinical Rockall &
\end{tabular}

AUC, area under the receiver operating characteristic curve; CI, confidence interval; GI, gastrointestinal; ICU, intensive care unit; ns, non-significant; UGIB, upper gastrointestinal bleeding.

The AIMS65 was significantly better in predicting in-hospital mortality (Fig. 1), with an AIMS65 score $\geq 2$ leading to maximal sensitivity (95.3\%) and specificity (63.1\%). Analysis of the subset of patients with upper GI cancers confirmed that AIMS65 was better for in-hospital mortality prediction (Table 2). Similarly, it was better in predicting UGIB-related in-hospital mortality.

\section{F. Low-risk patients}

The GBS was significantly superior in identifying the lowrisk group at admission compared with the other score systems (Table 2).

The specificity and sensitivity of GBS were $100 \%$ and 5.8\%, respectively, when the cut-off value for the selection of low-risk patients was set at 0 . When the cut-off value was set at 2 , the 


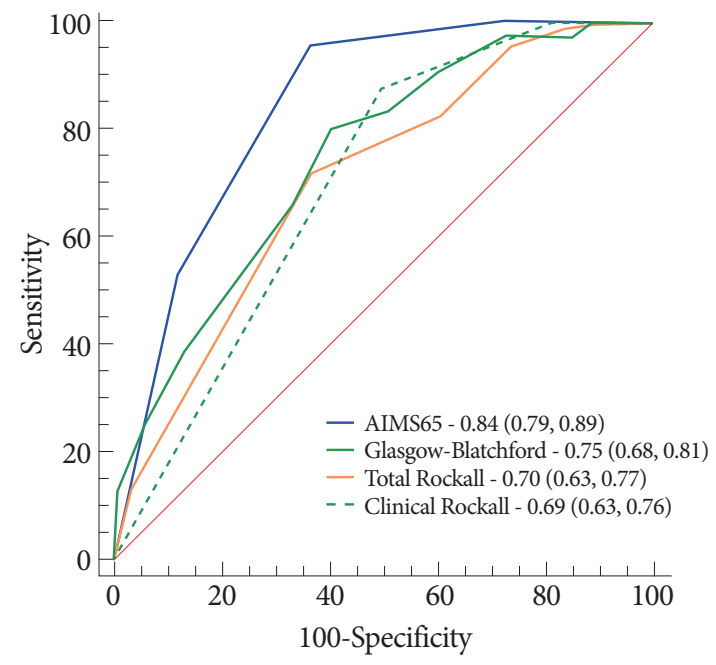

Fig. 1. Prediction of in-hospital mortality. The AIMS65 was superior to the other scoring systems.

specificity remained at $100 \%$, while the sensitivity increased to $23.5 \%$. The number of patients considered as low-risk increased from $1 \%$ to $5 \%$ when the cut-off value changed from 0 to 2, without causing high-risk patients to be discharged incorrectly (Fig. 2). Comparatively, an AIMS65 of 0 erroneously classified 20 high-risk patients who had major adverse events after bleeding (rebleeding, hemotransfusion, and/or hemostatic therapy) (Fig. 2), resulting in a sensitivity and specificity of $89.5 \%$ and $53 \%$, respectively.

\section{G. New scoring system}

All demographic and clinical variables were considered for inclusion in the new scoring system, which was developed using logistic regression analysis. The new scoring system considers the presence of a tumor in the upper GI tract; history of hematemesis, melena, liver disease; and the hemoglobin and international normalized ratio (INR) levels (Fig. 3). In the first step (z calculation in Fig. 3), ln is the natural logarithm of hemoglobin. This value is then converted into the final score ( $p$-value) with a value ranging from 0 to 100 (Fig. 3). When reporting, the values presented in the formula were not rounded off to remove the impact of the conversion on the 0-100 scale.

This scoring system had an AUC of 0.74 (CI: 0.67, 0.80) in predicting hemostatic therapy at admission, and comparisons revealed its significant superiority to the AIMS65, GBS, and clinical RS (Table 2 and Fig. 4).

A sensitivity, specificity, positive predictive value, and negative predictive value of $21 \%, 97 \%, 90 \%$, and $51 \%$, respectively, were observed with a score of 78.7 or higher. Therefore, this
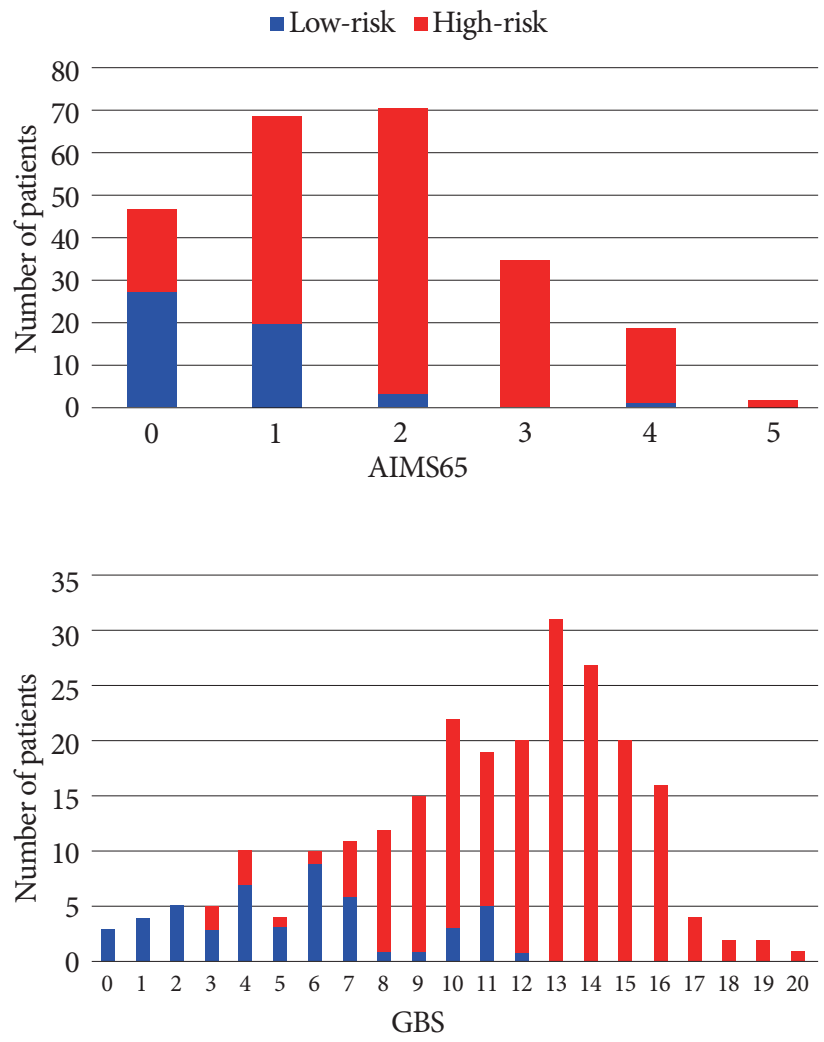

Fig. 2. Identification of low-/high-risk groups among cancer patients with upper gastrointestinal bleeding by score. GBS, Glasgow-Blatchford score.

$\mathrm{z}=2.7626+(0.5817$ if tumor in upper GI tract $)+(0.732$ if history of hematemesis $)+(0.6965$ if melena present $)+(0.4703$ if liver disease $)$ - $(1.2124 \times$ In (hemoglobin $))-\left(1.8816 \times(1 / \mathrm{INR})^{2}\right)$

$$
p=\left[\frac{\mathrm{e}^{\mathrm{z}}}{1+\mathrm{e}^{\mathrm{z}}}\right] \times 100
$$

Fig. 3. The new scoring system for predicting the need for hemostatic therapy at admission after upper gastrointestinal bleeding in cancer patients. In is the natural logarithm of hemoglobin. Using the formula, the value of $z$ is then converted into a score with values ranging from 0 to 100 . GI, gastrointestinal; INR, international normalized ratio.

score value may be used to detect early the patients at risk for hemostatic therapy. Additionally, a sensitivity, specificity, and positive and negative predictive values of $97 \%, 21 \%, 59 \%$, and $85 \%$, respectively, were observed with a score value of 23.5 or lower, which may be chosen as a cut-off to forego the need of hemostatic therapy (Fig. 5). 


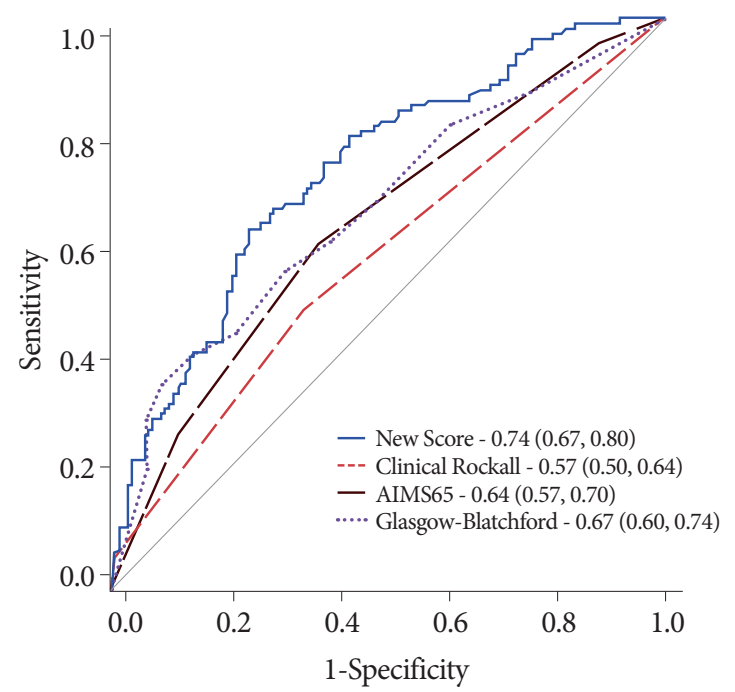

Fig. 4. Comparison of the scores for predicting hemostatic therapy in patients with cancer. The predictive accuracy of this newly developed score is significantly better than that of the clinical Rockall score $(p<0.001)$, AlMS65 $(p=0.001)$, and the Glasgow-Blatchford score $(p=0.027)$.

\section{DISCUSSION}

In patients with cancer who present with an acute episode of UGIB confirmed via EGD, we have shown that either primary cancer or metastasis was the source of bleeding in $40.1 \%$ of patients. Among patients with a known upper GI cancer, the likelihood of tumor bleeding was even higher (83\%). These findings were in accordance with our previous retrospective publication conducted in a different group of patients. ${ }^{1}$

As cancer patients pose unique challenges when presenting with UGIB, an appropriate risk stratification leading to effective treatment can be life-saving. Our findings revealed that all the scoring systems had comparable abilities in prognosticating all pertinent outcomes of UGIB among cancer patients.

The superior performance of AIMS65 in predicting ICU admission in our study was expected as Robertson et al. ${ }^{17}$ had previously reported its superior accuracy in predicting ICU admission among the general population with UGIB compared to the other scoring systems. Our results showed that the GBS was significantly better in predicting blood transfusion, validating the applicability of previously published findings in a general population with UGIB among cancer patients. ${ }^{10,17,18}$

Currently, there is no consensus on the optimal endoscopic therapy for patients with tumoral bleeding. In our previous experience, rebleeding and mortality were similar in patients

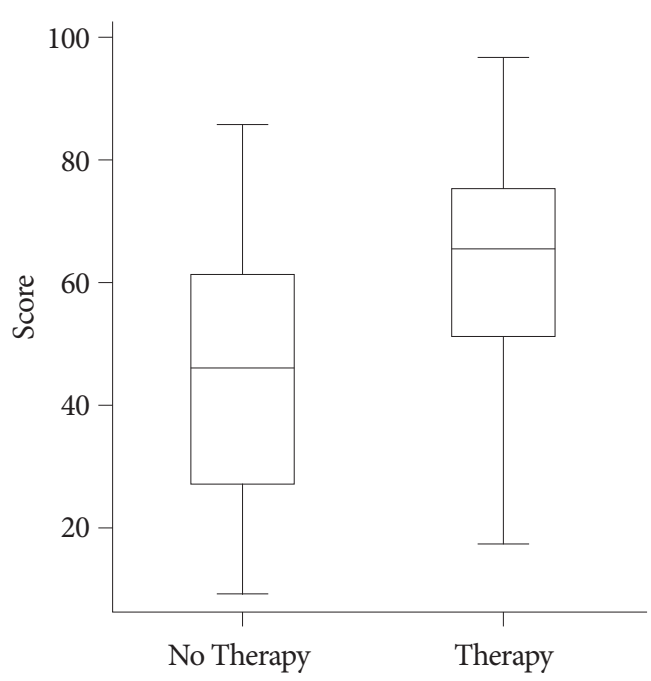

Fig. 5. The box plot of cancer patients with upper gastrointestinal bleeding demonstrated that the patients who needed hemostatic therapy had significantly higher scores in the new scoring system compared with those who did not require hemostatic therapy $(p<0.001)$. Boxes represent interquartile ranges with bars representing minimum to maximum.

with tumoral GI bleeding, regardless of whether they received endoscopic therapy or not. ${ }^{1,11}$ Patients with uncontrolled tumoral bleeding should be referred for inpatient hemostatic radiotherapy, angiographic embolization, or surgery. ${ }^{19}$ The Hemospray $^{\circledR}$ (Cook Medical, Bloomington, IN, USA) was introduced as a hemostatic powder for endoscopic bleeding control in cases of UGIB. Chen et al. ${ }^{20}$ reported its use in five patients with tumoral GI bleeding and found that all experienced immediate bleeding control, and only one case had rebleeding. Shin et al. ${ }^{21}$ also reported immediate bleeding control in all patients with the use of the hemostatic powder in a study with 41 consecutive patients with tumoral UGIB. Although a hemostatic powder seems to be a safe and reliable option for hemostasis of tumoral bleeding, it was not available yet in our institution during the period of this study.

In a study involving the general population with UGIB, Stanley et al. ${ }^{22}$ showed that GBS predicted more accurately the need for endoscopic therapy compared to AIMS65 and the clinical RS. In contrast, our findings revealed that all the scores had poor discrimination for pre-endoscopic prediction of hemostatic therapy among cancer patients. Kim et al. ${ }^{12}$ reported in a study with 357 cases of inoperable gastric cancer that the total RS was better than GBS and clinical RS for predicting urgent hemostatic therapy from tumor bleeding. However, the total RS has limitations in predicting hemostatic therapy 
because it only can be calculated at the time of diagnostic endoscopy. Based on these findings, a score that could select at admission the group of patients with cancer and UGIB who are more likely to need hemostatic therapy may provide better clinical results. Therefore, we created a new scoring system to be used at admission using data that are commonly assessed at initial evaluation to select the patients with cancer and UGIB who are at risk of requiring hemostatic therapy. Our results demonstrated that the new scoring system performed significantly better than the other scores in predicting hemostatic therapy. Although the process of obtaining the score value is complex, it contributed to $23.4 \%$ of this population of patients with cancer and UGIB (12.3\% with score $>78.7$ plus $11.1 \%$ with score <23.5).

The poor performances of all the scoring systems in predicting rebleeding observed in our study may point to the relative inadequacy of hemostatic procedures in patients with tumoral bleeding. The data on predicting rebleeding in a general population with UGIB are heterogeneous. Some studies have confirmed the poor performance of these score systems, while some have reported that GBS and RS performed better. ${ }^{17,18,22}$

In contrast with the overall mortality of UGIB in the general population of approximately $5-10 \%$, the 30 -day mortality of cancer patients with UGIB was $34.1 \%{ }^{23}$ Interestingly, the 30 day mortality rate was similar between the cases of tumor and non-tumor bleeding ( $38.1 \%$ vs. $31.9 \%$, respectively; $p=0.46)$. Therefore, a bleeding episode may represent the end stage of the malignant disease, regardless of the etiology of the source of bleeding.

Compared to the other scores, the AIMS65 best predicted in-hospital mortality. It also best predicted the UGIB-related in-hospital mortality and in-hospital mortality in cases with upper GI cancers. Comparatively, previous publications have already reported the superiority of AIMS65 in predicting in-hospital mortality for the general population with UGIB. ${ }^{10,17,24}$

The low-risk group can safely be treated as an outpatient, and in our study, this group was composed of the patients who did not receive blood transfusion, hemostatic therapy, and did not with present rebleeding or mortality during the 30-day follow up. Among the patients with cancer and UGIB, GBS was better than the other scores in identifying the low-risk group at admission. In addition, the GBS with a cut-off value $\leq 2$ can increase its sensitivity without affecting its high specificity. Similarly, Ahn et al. ${ }^{13}$ demonstrated that the GBS better predicted hospital-based interventions in patients with cancer, and the score performance was not compromised by the etiology of the bleeding. Similarly, Ahn et al. ${ }^{13}$ also reported that the cut-off $\leq 2$ in GBS had a higher accuracy.

There were limitations to this study. First, this was a sin- gle-center study, which limited our ability to generalize the results to similar populations in other institutions. Second, we included a heterogeneous population of cancer patients. However, stratified analysis in patients with primary cancer within and outside the GI tract revealed similar results. Third, there was no validation cohort for the new scoring system.

However, our study had its strengths as well. First, we prospectively collected detailed clinical information and hemostasis techniques and outcomes for each patient with a low dropout rate (6.2\%). Second, the Cancer Institute of the University of São Paulo is a 23-floor tertiary teaching hospital with more than 700 beds and a high number of referred patients from the country, enabling the enrollment of more than 200 cancer patients with UGIB over 1 year.

With the advent of novel oncologic and surgical therapies, the overall prognosis of some cancers has improved over the years. ${ }^{25}$ For some patients with cancer, UGIB may represent the final event in the natural history of the oncologic condition. However, for many of them, it may just be an adverse event of oncological therapy or disease. In fact, in our cohort, the 30-day mortality was around $30 \%$, indicating that the majority survived. Therefore, a UGIB represents a severe event in patients with cancer, and intensive care with dedicated therapies is needed to improve the outcomes.

In conclusion, the AIMS65 better predicted ICU admission and in-hospital mortality in cancer patients with UGIB, while GBS better predicted blood transfusion and the low-risk group who may be safely managed as outpatients. All the scores poorly predicted rebleeding and hemostatic therapy. Given the lack of score that superiorly and accurately predicted hemostatic therapy at admission in cancer patients with UGIB, we developed a new scoring system, which had a fair performance and was superior to the other scores. Further studies are necessary to validate this new scoring system tailored for this patient population.

Conflicts of Interest

The authors have no potential conflicts of interest.

Funding

None.

Author Contributions

Conceptualization: Matheus C. Franco, Bruno C. Martins, Vipul Jairath, John Vargo, Alan Barkun, Fauze Maluf-Filho

Data curation: MCF

Formal analysis: MCF, Sunguk Jang, BCM, Tyler Stevens, VJ, Rocio Lopez, AB, FMF

Resources: SJ, TS, RL, JV, FMF

Supervision: SJ, BCM, AB, FMF

Writing-original draft: MCF

Writing-review \& editing: SJ, BCM, TS, JV, AB, FMF 
ORCID

$\begin{array}{ll}\text { Matheus Cavalcante Franco } & \text { https://orcid.org/0000-0003-0525-8003 } \\ \text { Sunguk Jang } & \text { https://orcid.org/0000-0001-9837-0322 } \\ \text { Bruno Costa Martins } & \text { https://orcid.org/0000-0002-0282-0753 } \\ \text { Tyler Stevens } & \text { https://orcid.org/0000-0002-4950-3229 } \\ \text { Vipul Jairath } & \text { https://orcid.org/0000-0002-1092-0033 } \\ \text { Rocio Lopez } & \text { https://orcid.org/0000-0002-4319-420X } \\ \text { John J Vargo } & \text { https://orcid.org/0000-0001-7638-5283 } \\ \text { Alan Barkun } & \text { https://orcid.org/0000-0002-1798-5526 } \\ \text { Fauze Maluf-Filho } & \text { https://orcid.org/0000-0001-8875-420X }\end{array}$

\section{REFERENCES}

1. Maluf-Filho F, Martins BC, de Lima MS, et al. Etiology, endoscopic management and mortality of upper gastrointestinal bleeding in patients with cancer. United European Gastroenterol J 2013;1:60-67.

2. Targownik LE, Nabalamba A. Trends in management and outcomes of acute nonvariceal upper gastrointestinal bleeding: 1993-2003. Clin Gastroenterol Hepatol 2006;4:1459-1466.

3. Garcia-Tsao G, Bosch J. Management of varices and variceal hemorrhage in cirrhosis. $\mathrm{N}$ Engl J Med 2010;362:823-832.

4. Franco MC, Nakao FS, Rodrigues R, Maluf-Filho F, Paulo GA de, Libera ED. Proposal of a clinical care pathway for the management of acute upper gastrointestinal bleeding. Arq Gastroenterol 2015;52:283-292.

5. Barkun AN, Almadi M, Kuipers EJ, et al. Management of nonvariceal upper gastrointestinal bleeding: guideline recommendations from the International Consensus Group. Ann Intern Med 2019;171:805-822.

6. Rockall TA, Logan RF, Devlin HB, Northfield TC. Risk assessment after acute upper gastrointestinal haemorrhage. Gut 1996;38:316-321.

7. Vreeburg EM, Terwee CB, Snel P, et al. Validation of the Rockall risk scoring system in upper gastrointestinal bleeding. Gut 1999;44:331-335.

8. Blatchford O, Murray WR, Blatchford M. A risk score to predict need for treatment for upper-gastrointestinal haemorrhage. Lancet 2000; 356:1318-1321.

9. Saltzman JR, Tabak YP, Hyett BH, Sun X, Travis AC, Johannes RS. A simple risk score accurately predicts in-hospital mortality, length of stay, and cost in acute upper GI bleeding. Gastrointest Endosc 2011;74:12151224 .

10. Hyett BH, Abougergi MS, Charpentier JP, et al. The AIMS65 score compared with the Glasgow-Blatchford score in predicting outcomes in upper GI bleeding. Gastrointest Endosc 2013;77:551-557.

11. Martins BC, Wodak S, Gusmon CC, et al. Argon plasma coagulation for the endoscopic treatment of gastrointestinal tumor bleeding: a retrospective comparison with a non-treated historical cohort. United Euro- pean Gastroenterol J 2016;4:49-54.

12. Kim YI, Choi IJ, Lee JY, Kim CG, Kim HK, Park YL. Comparison of the performance of risk scoring systems for tumor bleeding in patients with inoperable gastric cancer. Endoscopy 2020;52:359-367.

13. Ahn S, Lim KS, Lee YS, Lee JL. Blatchford score is a useful tool for predicting the need for intervention in cancer patients with upper gastrointestinal bleeding. J Gastroenterol Hepatol 2013;28:1288-1294.

14. Laine L, Spiegel B, Rostom A, et al. Methodology for randomized trials of patients with nonvariceal upper gastrointestinal bleeding: recommendations from an international consensus conference. Am J Gastroenterol 2010;105:540-550.

15. DeLong ER, DeLong DM, Clarke-Pearson DL. Comparing the areas under two or more correlated receiver operating characteristic curves: a nonparametric approach. Biometrics 1988;44:837-845.

16. Youden WJ. Index for rating diagnostic tests. Cancer 1950;3:32-35.

17. Robertson M, Majumdar A, Boyapati R, et al. Risk stratification in acute upper GI bleeding: comparison of the AIMS65 score with the Glasgow-Blatchford and Rockall scoring systems. Gastrointest Endosc 2016;83:1151-1160

18. Martínez-Cara JG, Jiménez-Rosales R, Úbeda-Muñoz M, de Hierro ML, de Teresa J, Redondo-Cerezo E. Comparison of AIMS65, GlasgowBlatchford score, and Rockall score in a European series of patients with upper gastrointestinal bleeding: performance when predicting in-hospital and delayed mortality. United European Gastroenterol J 2016;4:371-379.

19. Hwang JH, Fisher DA, Ben-Menachem T, et al. The role of endoscopy in the management of acute non-variceal upper GI bleeding. Gastrointest Endosc 2012;75:1132-1138.

20. Chen YI, Barkun AN, Soulellis C, Mayrand S, Ghali P. Use of the endoscopically applied hemostatic powder TC-325 in cancer-related upper GI hemorrhage: preliminary experience (with video). Gastrointest Endosc 2012;75:1278-1281.

21. Shin J, Cha B, Park JS, et al. Efficacy of a novel hemostatic adhesive powder in patients with upper gastrointestinal tumor bleeding. BMC Gastroenterol 2021;21:40.

22. Stanley AJ, Laine L, Dalton HR, et al. Comparison of risk scoring systems for patients presenting with upper gastrointestinal bleeding: international multicentre prospective study. BMJ 2017;356:16432.

23. Abougergi MS, Charpentier JP, Bethea E, et al. A prospective, multicenter study of the AIMS65 score compared with the Glasgow-Blatchford score in predicting upper gastrointestinal hemorrhage outcomes. J Clin Gastroenterol 2016;50:464-469.

24. van Leerdam ME. Epidemiology of acute upper gastrointestinal bleeding. Best Pract Res Clin Gastroenterol 2008;22:209-224.

25. Maluf-Filho F. Predicting clinical outcomes in patients with bleeding from gastric cancer: novel tools are needed to nail it! Endoscopy 2020;52:332-333. 